\title{
Towards Brain-Computer Music Interfaces: Progress and Challenges
}

\author{
Eduardo R. Miranda, Simon Durrant and Torsten Anders
}

\begin{abstract}
Brain-Computer Music Interface (BCMI) is a new research area that is emerging at the cross roads of neurobiology, engineering sciences and music. This research involves three major challenging problems: the extraction of meaningful control information from signals emanating directly from the brain, the design of generative music techniques that respond to such information, and the training of subjects to use the system. We have implemented a proof-of-concept BCMI system that is able to use electroencephalogram information to generate music online. Ongoing research informed by a better understanding of brain activity associated with music cognition, and the development of new tools and techniques for implementing braincontrolled generative music systems offer a bright future for the development of BCMI.
\end{abstract}

Index Terms - Biomedical engineering, electroencephalogram, functional magnetic resonance imaging, music.

\section{INTRODUCTION}

$\mathrm{G}$ enerally speaking, a Brain-Computer Interface (BCI) is a system that allows one to interact with a computing device by means of signals emanating directly from the brain. Basically, there are two ways to read brain signals: invasive and non-invasive. Whereas invasive methods require the placement of sensors connected to the brain inside the skull, non-invasive methods use sensors that can read brain signals from the outside the skull. The most current viable non-invasive method for BCI is the electroencephalogram, or EEG.

Research into Brain-Computer Music Interface (BCMI) is an emerging topic at the cross roads of neurobiology, engineering sciences and music. Whilst developments in electronic technologies take place exponentially in health care and within the music industry, there has been little development addressing the well-being of people within the health and education sectors. BCMI research may open up many possibilities, in particularly for people with special needs: as a recreational device for people with disabilities, music therapy, and as an instrument for concert performance and composition. Currently, research into BCMI involves three major challenging problems on their own right, namely: a) the

Manuscript received July 25, 2008. This work was supported by EPSRC, UK, under the "Learning the Structure of Music" project (Le StruM), grant EPD063612-1.

E. R. Miranda, S. Durrant and T. Anders are with the Interdisciplinary Centre for Computer Music Research (ICCMR), University of Plymouth, United Kingdom. E. R. Miranda is the corresponding author (+44 (0)1752 586255, e-mails: \{eduardo.miranda, simon.durrant, torsten.anders\}@plymouth.ac.uk) extraction of meaningful control information from the EEG, b) the design of generative music techniques that respond to EEG information and c) the training of subjects to use the system. This paper focuses on the first two challenges. It begins with a brief historical account of research into BCMI and approaches to systems design. Then it introduces the BCMI-Piano, a proof-of-concept system that uses EEG information to generate new pieces music on-line ("real-time"), followed by a brief discussion on its limitations and challenges for improvement. Next, we present a brain imaging-based experiment aimed at identifying neural correlates of tonal processing. Finally, we propose a generative music approach based on constraint satisfaction techniques as a way forward to generate music with a BCMI. An example of a generative music system inspired by the results of the experiment is also introduced.

\section{A. Brief Historical Account}

Human brainwaves were first measured in the $1920 \mathrm{~s}$, in Germany, by Hans Berger. He termed these measured brain electrical signals the electroencephalogram (literally "brain electricity writing"). Berger first published his brainwave results in 1929 [1] but was not until 1969 that Pierre Gloor translated the article into English [2]. In the early 1970s, in the USA, Jacques Vidal worked on the first attempt towards a BCI system [3]. Many attempts followed with various degrees of success. But it was in the early 1990s that the field started to make significant progress; e.g., Jonathan Wolpaw and colleagues developed a BCI to allow some control of a computer cursor using aspects of EEG's alpha rhythms (i.e., frequency components between $8 \mathrm{~Hz}$ and $13 \mathrm{~Hz}$ ) [4].

With respect to BCI for music, as early as 1934, a paper in the journal Brain had reported a method to listen to the EEG [5]. But it is now generally accepted that it was Alvin Lucier who composed the first musical piece using EEG in the mid of the 1960s: Music for Solo Performer [6]. He placed electrodes on his own scalp, amplified the signals, and relayed them through loudspeakers that were "directly coupled to percussion instruments, including large gongs, cymbals, tympani, metal ashcans, cardboard boxes, bass and snare drums..." [7]. The low frequency vibrations emitted by the loudspeakers set the surfaces and membranes of the percussion instruments into vibration. In the early 1970s David Rosenboom began systematic research into the potential of EEG to generate music [8]. He explored the hypothesis that it might be possible to detect certain aspects of our musical experience in the EEG signal. This was an important step for BCMI research as Rosenboom pushed the practice beyond the direct sonification of EEG signals, towards the notion of 
digging for potentially useful information in the EEG to make music with. In 1990 he introduced a musical system whose parameters were driven by EEG components believed to be associated with shifts of the performer's selective attention [9]. Thirteen years later, Eduardo R. Miranda and colleagues reported new experiments and techniques to enhance the EEG signal and train the computer to identify EEG patterns associated with different cognitive musical tasks [10]. Subsequently, Miranda implemented the BCMI-Piano system [11], which is briefly introduced later in this paper.

\section{B. Approaches to BCI Design}

It is possible to identify three categories of BCI systems: user oriented, computer oriented and mutually oriented. User oriented systems are BCI systems where the computer adapts to the user. Metaphorically speaking, these systems attempt to "read the mind" of the user to control a device. For example, Anderson and Sijercic reported on the development of a BCI that learns to associate specific EEG patterns from a subject with commands for navigating a wheelchair [12]. Computer oriented are $\mathrm{BCI}$ systems where the user adapts to the computer. These systems rely on the capacity of the users to learn to control specific aspects of their EEG, affording them the ability to exert some control over events in their environments. Examples have been shown where subjects learn to steer their EEG to select letters for writing words on a computer screen [13]. Finally, mutually oriented are BCI systems combining the functionalities of both categories, where the user and the computer adapt to each other. The combined use of mental task pattern classification and biofeedback assisted on-line learning allows the computer and the user to adapt. Prototype systems to move a cursor on the computer screen have been developed in this fashion [14].

The great majority of those who have attempted to employ EEG as part of a music controller have done so by associating certain EEG characteristics, such as the power of the EEG alpha waveband (also referred to as alpha rhythms) to specific musical actions. These are essentially computer oriented systems, as they require the user to learn to control their EEG in certain ways.

\section{THE BCMI-PIANO SYSTEM}

The BCMI-Piano falls into the category of BCI computer oriented systems. The system is programmed to look for information in the EEG signal and match the findings with assigned generative musical processes corresponding to different musical styles. The BCI-Piano is composed of four main modules: sensing, analysis, music engine and performance.

The EEG is sensed with 7 pairs of gold EEG electrodes on the scalp (bipolar montage), as follows: G-Fz, F7-F3, T3-C3, O1-P3, O2-P4, T4-C4, F8-F4 [15]. In this particular case, we were not looking for specific signals emanating from different cortical sites. The objective here is to sense the EEG over the whole surface of the cortex. The electrodes are plugged into a biosignal amplifier and a real-time acquisition system manufactured by Guger Technologies, Austria. The analysis module generates two streams of control parameters. One stream contains information about the most prominent frequency band in the signal and is used by the music engine module to generate the music. In the current version, the music engine module composes two different styles of music, depending on whether the EEG indicates salient alpha rhythms (between $8 \mathrm{~Hz}$ and $13 \mathrm{~Hz}$ ) or beta rhythms (between $14 \mathrm{~Hz}$ and $33 \mathrm{~Hz}$ ). The other stream contains information about the complexity of the signal, extracted using Hjorth signal complexity analysis [16]. The music engine uses this information to control the tempo and the loudness of the music.

The core of the music engine module is a set of generative music rules. Each rule produces a musical bar or half-bar. In a nutshell, the music engine works as follows: every time it has to produce a bar of music, it checks the power spectrum of the EEG at that moment and activates rules associated with the most prominent EEG rhythm in the signal. The system is initialized with a reference tempo (e.g., 120 beats per minute), which is constantly modulated by the results from the signal complexity analysis. The music engine sends out MIDI information to the performance module, which plays the music using a MIDI-enabled acoustic piano (Fig. 1).

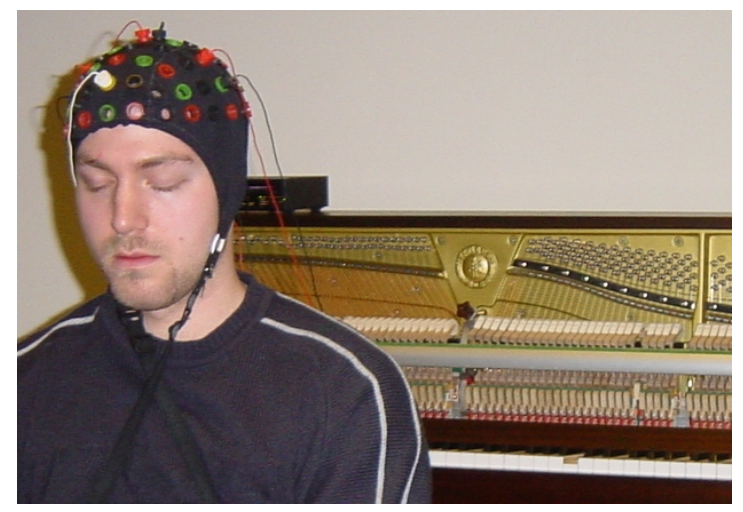

Fig. 1. The music is played on a MIDI-enabled acoustic piano. (Note: the electrodes montage in this photograph is not the same as the one described in the paper. This photo is from an earlier stage of the work.)

The music engine generates new music using rules extracted from given musical examples. It extracts sequencing rules from a corpus of music examples and creates a transition matrix representing the transition-logic of what-follows-what. New musical pieces in the style of the ones in the training corpus are generated by sequencing building blocks of music material (also extracted from the examples in the corpus) in a domino-like manner. Although this type of self-learning predictors of musical elements based on previous musical elements could be used for any type of musical element (such as musical note, chord, bar, phrase, section, and so on), we have focused here on short vertical slices of music such as a bar or half-bar. The predictive characteristics are determined by the chord (harmonic set of pitches, or pitch-class) and by the first melodic note following the melodic notes in those vertical slices of music. We created a simple method for generating musical phrases with a beginning and an end that can be determined by EEG information. The system can generate piano music that contains, for example, more Eric Satie-like elements when the spectrum of the subject's EEG 
contains salient alpha rhythms and more Beethoven-like elements when the spectrum of the EEG contains salient beta rhythms. A demonstration movie of the BCMI-Piano is available at ICCMR's website (Accessed 23 July 2008):

http://cmr.soc.plymouth.ac.uk/media/Tokyo_demo.mov>.

\section{MOVING FORWARDS}

\section{A. The Challenges}

In order to move research into BCMI forwards, two major challenges need to be addressed: a) discovery of meaningful musical information in brain signals for control beyond the standard EEG rhythms and $b$ ) design of powerful techniques and tools for implementing flexible and sophisticated on-line generative music systems. In order to the address the former we have started to perform a number of brain imaging experiments aimed at gaining a better understanding of brain correlates of music cognition, with a view on discovering patterns of brain activity suitable for BCMI control. In the following section we report on the results of an experiment on musical tonality. In order to address the second challenge we are devising systems for generative music based on constraint satisfaction programming techniques.

\section{B. fMRI Experiment: Neural Processing of Tonality}

Tonality is central to the experience of listening to tonal music, but to date there is no definitive evidence as to the neural substrate underlying it. Here we present a functional Magnetic Resonance Imaging (fMRI) study of tonality, focusing in particular on the difference in neural processing of tonal and atonal stimuli, and neural correlates of distance around the circle-of-fifths, which describes how close one key is to another.

Tonality describes a music theoretic concept [17] with perceptual reality [18]. It is concerned with the establishment of a sense of key, which in turn defines a series of expectations and interpretations of musical tones. Within Western tonal music, the octave is divided into twelve equal semitones, seven of which are said to belong to the scale of any given key. Within these seven tones, the first (lowest) is the most fundamental, and the one that the key is named after. Other tones (in particular three, four and five) are also regarded as important. A sense of key can be established by a monotonic (single) melodic line, with harmony implied, but can also have that harmony explicitly created in the form of chord progressions (homophony). Tonality also defines clear expectations, with the chord built on the first tone (or degree) again taking priority and the chords based on the fourth and fifth degrees also particular important because their constituent members are the only ones whose constituent tones are entirely taken from the seven tones of the original scale, and occurring with greater frequency than other chords. The chord based on the fifth degree is followed the majority of the time by the chord based on the first degree (in musical jargon, a dominant-tonic progression). This special relationship also extends to different keys, with the keys based on the fourth and fifth degrees of a scale being closest to an existing key (based on the first degree of the scale) by virtue of sharing all but one scale tone with that key. This gives rise to the circleof-fifths [19] where a change (or modulation) from one key to another is typically to one of these other keys that are close in this way. Hence we can define the closeness of keys based on their proximity in the circle of fifths, with keys whose first degree scale tones are a fifth apart sharing most of their scale tones, and being perceived as closest to each other.

\section{Materials and Methods}

Sixteen subjects ( 9 female, 7 male; age 19-31; right handed; normal hearing) gave informed consent to take part in the experiment, which was approved by the Ethics Committee of the University of Magdeburg and Leibniz Institute for Neurobiology, Germany. None had received any formal musical education and none had absolute pitch. Musical sequences were $8 \mathrm{~s}$ long and consisted of 16 isochronous piano sounds lasting $500 \mathrm{~ms}$; each sound consisted of four simultaneous tones forming a chord recognized in Western tonal music theory (Fig. 3). Three of these sequences were ordered into twenty four groups with no gaps between sequences and groups. The first sequence in each group (initial condition) was always tonal presented in the home key of $\mathrm{C}$ major. The second was also tonal and could either be in F\# major (distant key condition), in G major (close key condition), or in $\mathrm{C}$ major (same key condition). The third sequence in each group was always atonal (atonal condition), which reset the listener's sense of key. The stimuli were ordered such that all tonal stimuli were used an equal number of times. The conditions appeared in all permutations equally in order to control for order effects.

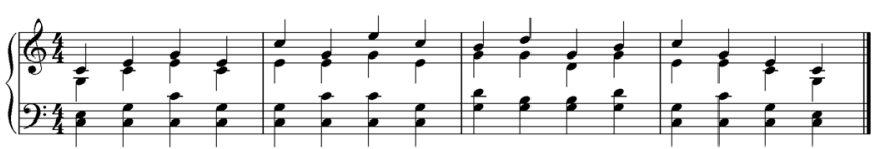

Fig. 3. Tonal stimuli in the key of $\mathrm{C}$ major, which constitute the initial and same conditions.

TABLE I

ACTIVATIONS RELATED TO KEY CHANGES

\begin{tabular}{|l|c|c|c|c|}
\hline Anatomical Name & X & Y & Z & Cluster \\
\hline (1) Right Transverse Temporal Gyrus & 51 & -17 & 10 & 1023 \\
\hline (2) Right Insula & 36 & 17 & 13 & 948 \\
\hline (3) Right Lentiform Nucleous & 24 & -1 & 1 & 750 \\
\hline (4) Right Caudate & 14 & -4 & 22 & 1443 \\
\hline (5) Left Anterior Cingulate & -1 & 41 & 11 & 2574 \\
\hline (6) Left Superior Frontal Gyrus & -12 & 50 & 36 & 2241 \\
\hline (7) Left Transverse Temporal Gyrus & -51 & -18 & 11 & 981 \\
\hline
\end{tabular}

Anatomical results contrasting conditions with and without a key change. These active clusters preferentially favour key change stimuli. $\mathrm{X}, \mathrm{Y}$ and $\mathrm{Z}$ are Talairach coordinates for plotting scans onto a standard template after normalization of brain size and shape across the subjects.

The subjects were instructed to indicate any change from one key to another by clicking on the left button of a mouse, and a change towards a sequence with no key by clicking on the right button. Subjects were given an initial practice period in order to ensure that they understood the task. Functional volumes were collected at 3 Tesla using echo planar imaging ( $\mathrm{TE}=30 \mathrm{~ms}$; TR=2000ms; FA: $80 ; 32$ slices with $3 \times 3 \times 3 \mathrm{~mm}$ resolution, 606 volumes). Data processing and analysis was conducted using BrainVoyager QX 1.9 (Brain Innovation 
B.V., The Netherlands).

In short, the group analysis revealed a cluster of fMRI activation around the auditory cortex (especially in the left hemisphere) showing a systematic increase in BOLD (BloodOxygen-Level dependent) amplitude with increasing distance in key.
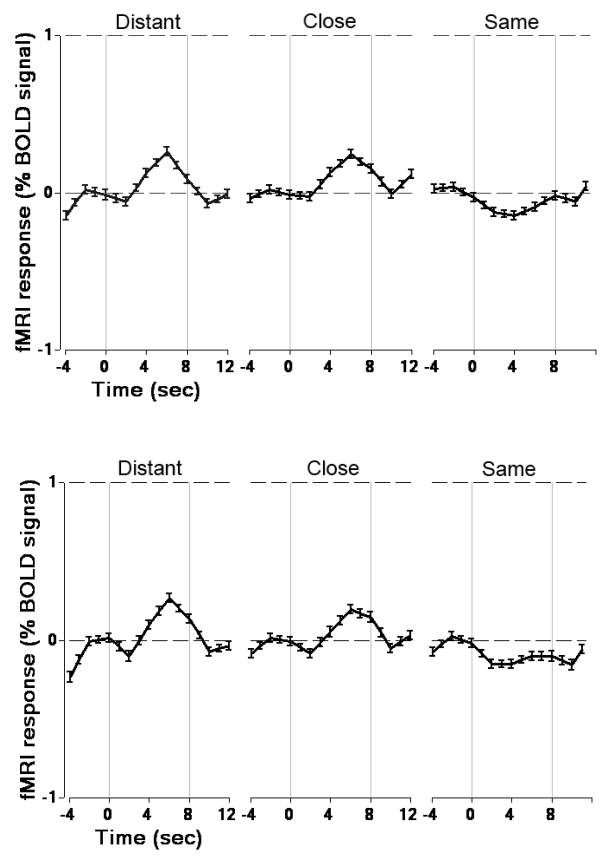

Fig. 4. Activation curves in left (top graph) and right (bottom graph) transverse temporal gyri for distant condition (plot on the left side), close condition (plot in the middle) and same condition (plot on the right side).

We have found a number of active neural clusters associated with the processing of tonality, which represent a diverse network of activation - some of these clusters are shown in Table 1 and Fig. 5. The results will be discussed in more detail in a forthcoming paper [20]. Here we focus on two particularly notable results. First is the strong presence of medial structures, in particular cingulate cortex (label 5 in Fig. 5 and Table I) and caudate nucleus (label 4 in Fig. 5 and Table I) in response to key changes. Second is the bilateral activation of the transverse temporal gyrus (labels 1 and 7 in Fig. 5 and Table I; also known as Heschl's gyrus), which contains the primary auditory cortex, for key changes. The activation curves for the bilateral activation of the transverse temporal gyrus show strongest activity for the distant key changes, slightly less, but still significant activity for the close key changes, and much less activity for no key changes (Fig. 4). It should be emphasized that this occurred across a variety of different stimuli, all of equal amplitude and with very similar basic auditory features, such as envelope and broad spectral content. Both left and right transverse temporal gyri showed very similar response curves (Fig. 4), highlighting the robust nature of these results. They suggest that these areas may not be limited to low-level individual pitch - or single note processing as commonly thought, but also be involved in some higher-order sequence processing. This is significant for our research as it indicates fairly well defined potential sources of control information for a BCMI, associated with tonality and modulation.
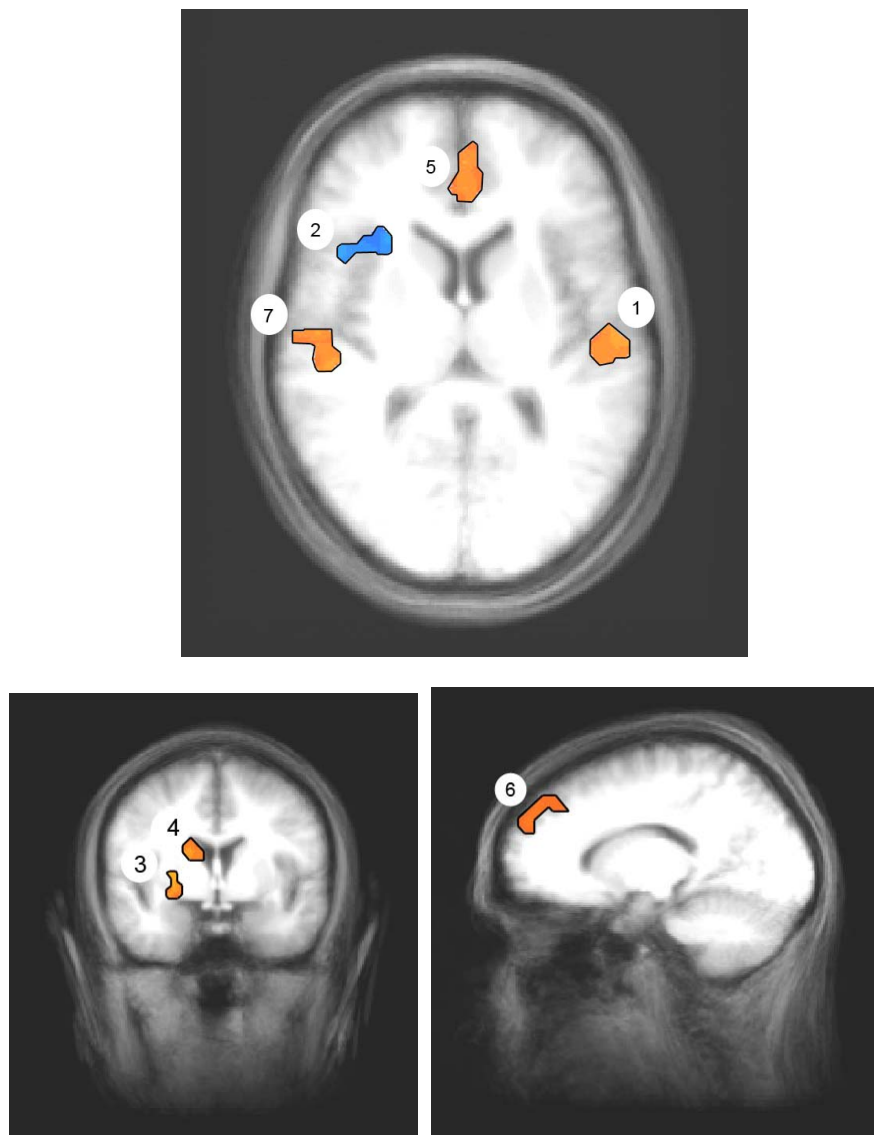

Fig. 5: Examples of clusters of activation for the contrast distant and close key vs. same key, including bilateral activation of transverse temporal gyrus for which the activation curves are shown in Fig. 4.

\section{Generative Music by Constraints Programming}

A constraint satisfaction problem (CSP) consists of a set of variables and mathematical relations between them, which are called constraints. A CSP usually presents a combinatorial problem and a constraint solver may find one or more solutions. We are developing a highly generic music constraint system, Strashella [21] where users can define a wide range of musical CSPs, including rhythmic, harmonic, melodic and contrapuntal problems. The user can freely apply different constraints to arbitrary sets of "score objects" (i.e., musical parameters, such as notes, rhythms, etc.). In addition to the definition of constraints, the user can also define convenient constraint application mechanisms. More information about the inner workings of Strashella can be found in [22] and [23].

We have used Strashella to implement an illustrative example of a generative music system embedding the findings of the experiment described in the previous section: it generates sequences of four-bar homophonic chord progressions on-line (Fig. 5). The input to the system is a stream of pairs of hypothetical EEG analysis data, which controls higher-level aspects of a forthcoming chord progression. The first value of the pair specifies whether a progression should form a cadence, which clearly expresses a specific key (cadence progression), or a chord sequence 
without any recognizable key (key-free progression). Additionally, if the next progression is a cadence progression, then the key of the cadence is specified by the second value of the pair.
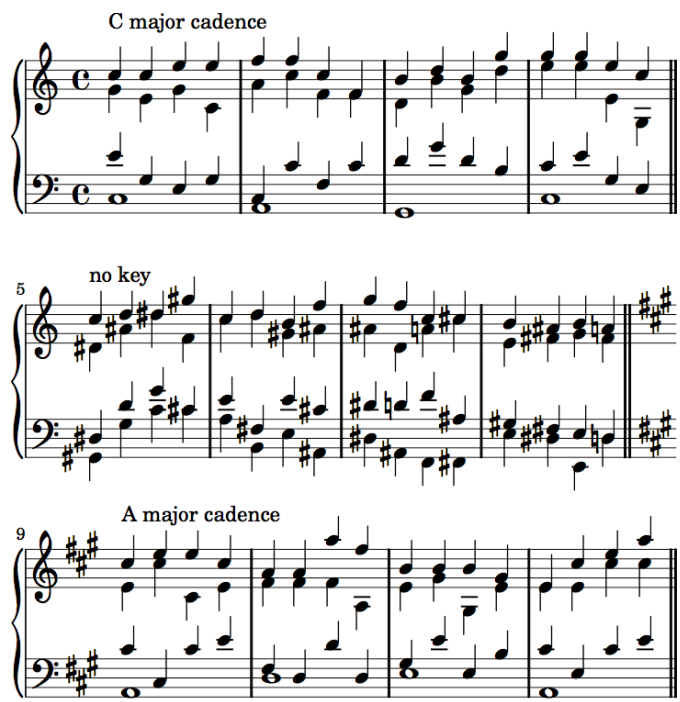

Fig. 5. Extract from a sequence of chord progressions generated by our illustrative example of a constraints-based generative system. In this case the system produced a sequence in $\mathrm{C}$ major, followed by a sequence in no particular key and then a sequence in A major.

Each progression consists in $n$ major or minor chords (in the example $n=16$ ), but different compositional rules are applied to cadence and key-free progressions. For instance, in the case of a cadence, the underlying harmonic rhythm is slower than the actual chords (e.g., one harmony per bar), and all chords must fall in a given major scale. The progression starts and ends in the tonic, and intermediate root progressions are restricted by Schoenberg's rules for tonal harmony [24]. For a key-free progression, rules enforce that all 12 chromatic pitch classes are used. For example, the roots of consecutive chords must differ and the set of all roots in the progression must express the chromatic total. Also, melodic intervals must not exceed an octave. A custom dynamic variable ordering speeds up the search process by visiting harmony variables (the root and whether it is major or minor), then the pitch classes and finally the pitches themselves. The value ordering is randomized, so we always get different solutions.

\section{CONCLUSION}

The discovery of brain correlates of music cognition needs to be followed by a studies to establish how such information can be used for BCMI control and also whether subjects can be trained to produce them voluntarily. For instance, would subjects be able to learn produce bilateral activations of transverse temporal gyrus simply by imagining tonal progressions? And if so, would one be able to detect such information in the EEG? These and many other technical challenges need to be addressed in order to pave the way for future BCMI systems.

\section{ACKNOWLEDGMENT}

We would like to thank A Brechman and $\mathrm{H}$ Scheich at the
Leibniz Institute for Neurobiology, Magdeburg, Germany, for their assistance with the fMRI experiment and the opportunity to use their Siemens Trio 3T MRI scanner.

\section{REFERENCES}

[1] Berger, H. (1929), "Über Das Elektrenkephalogramm Des Menschen." Archiv für Psychiatrie und Nervenkrankheiten, 87(1929):527-70.

[2] Berger, H. (1969), "On the Electroencephalogram of Man", The Fourteen Original Reports on the Human Electroencephalogram, Electroencephalography and Clinical Neurophysiology, Supplement No. 28. Amsterdam: Elsevier.

[3] Vidal, J.J. (1973), "Toward Direct Brain-Computer Communication." Annual Review of Biophysics and Bioengineering, L. J. Mullins (Ed.) Annual Reviews Inc., pp. 157-80.

[4] Wolpaw, J., McFarland, D., Neat, G., Forneris. C. (1991), "An Eeg-Based Brain-Computer Interface for Cursor Control." Electroencephalogr Clin Neurophysiol 78(3):252-9.

[5] Adrian, E.D., and Matthews, B.H.C. (1934), "The Berger Rhythm : Potential Changes from the Occipital Lobes in Man." Brain, 57(4): 35585.

[6] Lucier, A. (1976), "Statement On: Music for Solo Performer", D. Rosenboom (Ed.), Biofeedback and the Arts, Results of Early Experiments. Vancouver: Aesthetic Research Center of Canada Publications.

[7] Lucier, A. (1980), Chambers. Middletown, Conn. : Wesleyan University Press.

[8] Rosenboom, D. (1990a), Extended Musical Interface with the Human Nervous System, Leonardo Monograph Series No. 1. Berkeley, California: International Society for the Arts, Science and Technology.

[9] Rosenboom, D. (1990b), "The Performing Brain". Computer Music Journal 14(1):48-65.

[10] Miranda, E.R., Sharman, K., Kilborn, K. and Duncan, A. (2003), "On Harnessing the Electroencephalogram for the Musical Braincap", Computer Music Journal, 27(2):80-102.

[11] Miranda, E. R. (2007). "Brain-Computer music interface for composition and performance". International Journal on Disability and Human Development 5(2):119-125.

[12] Anderson, C. and Sijercic, Z. (1996), "Classification of EEG signals from four subjects during five mental tasks", Solving Engineering Problems with Neural Networks: Proceedings of the Conference on Engineering Applications in Neural Networks (EANN'96), pp. 507-414.

[13] Birbaumer, N., Ghanayin, N., Hinterberger, T., Iversen, I., Kotchoubey, B., Kubler, A., Perelmeouter, J., Taub, E. and Flor, H. (1999), “A spelling device for the paralysed", Nature 398:297-298.

[14] Peters, B.O., Pfurtscheller, G. and Flyvberg, H. (1997), "Prompt recognition of brain states by their EEG signals", Theory in Biosciences 116:290-301.

[15] Misulis, K, E. (1997). Essentials of Clinical Neurophysiology. Boston (MA): Butterworth-Heinemann.

[16] Hjorth, B. (1970). "EEG analysis based on time series properties", Electroencephalography and Clinical Neurophysiology, 29:306-310.

[17] Piston, W., Devoto, M. (1987). Harmony, 5th ed. Norton, New York.

[18] Krumhansl, C. L. (1990). Cognitive Foundations of Musical Pitch. Oxford, Oxford University Press.

[19] Shepard, R. N. (1982). Structural representations of musical pitch. In: Deutsch, D. (Ed.), The Psychology of Music. Oxford, Oxford University Press, pp. 344-390.

[20] Durrant, S., Miranda, E. R., Brechmann, A. and Scheich, H. (2008). "An fMRI Study of Neural Correlates of Musical Tonality". (Submitted to a journal)

[21] Anders, T. and Miranda, E. R. (2008). "Higher-Order Constraint Applications for Music Constraint Programming", Proceedings of International Computer Music Conference - (ICMC2008), Belfast (UK).

[22] Anders, T. (2007). Composing Music by Composing Rules: Design and Usage of a Generic Music Constraint System. PhD Thesis, Queen's University Belfast, UK.

[23] Anders, T. and Miranda, E. R. (2008). "Constraint-Based Composition in Realtime", Proceedings of International Computer Music Conference (ICMC2008), Belfast (UK).

[24] Schoenberg, A. (1986). Harmonielehre. Wien: Universal Edition. $\left(7^{\text {th }}\right.$ Edition) 